\title{
Núcleos y ámbitos de investigación sobre adicciones en España a través del análisis de los enlaces bibliográficos en la Web of Science (2000-2013)
}

\section{Core research areas on addiction in Spain through the Web of Science bibliographic coupling analysis (2000-2013)}

\author{
Gregorio González-Alcaide*; Amador Calafat**; Elisardo Becoña****** \\ * Departamento de Historia de la Ciencia y Documentación, Universitat de València; ** European Institute of Studies on \\ Prevention (IREFREA), Palma de Mallorca; *** Departamento de Psicología Clínica y Psicobiología, Universidad de Santiago \\ de Compostela.
}

\section{Resumen}

En el presente estudio se han identificado los principales núcleos de la investigación española en el área de las adicciones a través del estudio de los enlaces bibliográficos, en las publicaciones bajo el epígrafe 'Substance abuse' de la Web of Science. Este procedimiento analítico determina la proximidad temático-intelectual de los documentos objeto de estudio a partir de la identificación de la bibliografía común compartida o citada simultáneamente. Se ha efectuado un análisis factorial y un análisis de redes para agrupar los documentos, representar gráficamente los núcleos de investigación existentes y analizar las interrelaciones entre los mismos. Se han identificado 30 núcleos de investigación. El alcohol constituye el objeto de atención de 17 núcleos y la cocaína tiene una destacada presencia en 6 núcleos. La heroína y los opiáceos únicamente están presentes como sustancias destacadas en 4 núcleos y el cannabis y el tabaco en otros dos núcleos para cada una de ellas. Se ha constatado la existencia de un importante grado de atomización en el área, con la existencia de numerosos núcleos de investigación pero con pocas conexiones entre sí, con un reducido número de documentos que recogen un conocimiento común compartido. Destaca también el elevado número de núcleos emergentes, reflejo de un estado incipiente de muchos de los temas de investigación. Se debe incidir en prácticas que fomenten el consenso científico y la cohesión de la disciplina, así como favorecer la consolidación de las principales líneas que den respuesta a los problemas sociales y desafíos de la investigación. Palabras Clave: bibliometría, adicciones, ámbitos de investigación, enlaces bibliográficos, estructura intelectual.

\begin{abstract}
The present study identifies the main Spanish core research areas in the area of addictions through the bibliographic coupling analysis of the publications at the Web of Science under the substance abuse heading. The bibliographic coupling methodology is the analytical procedure that determines the thematic-intellectual proximity of the documents under consideration through the identification of the shared or simultaneously cited bibliography by those documents. A factor analysis and network analysis have been carried out to cluster documents, graphically represent the existing core research areas, and analyse the interrelations between them. We have identified 30 core research areas. Alcohol is the topic of attention of 17 areas and cocaine has a strong presence in 6 . Heroin and opiates are only present as prominent substances in 4 areas and cannabis and tobacco in other two for each substance. It has been found that there is a significant degree of fragmentation in the area, with the existence of numerous research foci but with few connections with each other and few documents showing shared common knowledge. Also noteworthy is the large number of emerging research areas, reflecting an incipient stage in many of the research topics. Consideration must be placed in promoting scientific consensus and cohesion of the discipline as well as to encouraging the consolidation of main lines that respond to the social problems and research challenges.

Key Words: bibliometrics, addiction, research areas, bibliographic coupling, intellectual structure.
\end{abstract}




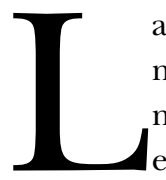

a investigación científica, como proceso de generación de nuevo conocimiento, parte del conocimiento preexistente sobre el tema objeto de estudio. Las referencias bibliográficas constituyen el mecanismo a través del cual el nuevo conocimiento se vincula con el conocimiento previo. El hecho de que un autor incluya una referencia bibliográfica a otro estudio, implica que ha utilizado ese documento para la realización de su trabajo y que considera que constituye el documento más importante entre todos los documentos posibles que ha podido citar. El estudio de las referencias bibliográficas recogidas en las publicaciones científicas de un área de conocimiento permite reconstruir el proceso intelectual de generación de nuevo conocimiento, identificando las áreas de conocimiento existentes y determinando el papel y la influencia desempeñada por las publicaciones en la literatura posterior (Culebras-Fernández, García de Lorenzo, Wanden-Nerghe, David y Sanz-Valero, 2008; Liu, 1993;).

Aunque existen algunos precedentes anteriores, el impulso y la institucionalización de los estudios basados en el análisis de las referencias bibliográficas incluidas en los documentos, se remonta a mediados de la década de 1950 y se puede personalizar en la figura de Eugene Garfield y en la creación de los índices de citas por parte del Institute for Scientific Information (ISI), cuyo desarrollo ha llegado hasta la actualidad a través de las bases de datos de la Web of Science de Thomson Reuters y más recientemente con la base de datos Scopus de Elsevier, que recogen, tanto la información bibliográfica que permite la identificación de los documentos, como las referencias bibliográficas citadas en los mismos. Aunque el interés inicial de los índices de citas era facilitar el proceso de recuperación de la información y optimizar el coste del acceso a la literatura científica, también contribuyeron de forma decisiva al estudio de los procesos de generación y difusión del conocimiento y a identificar las estructuras intelectuales subyacentes detrás de las interrelaciones establecidas entre los documentos a través de las referencias bibliográficas (Garfield, 1955).

Existen tres metodologías basadas en la identificación y el análisis de las referencias bibliográficas de los documentos con el propósito de analizar la estructura de la Ciencia y los procesos de difusión del conocimiento científico: los estudios de citación directa, los análisis de co-citas y el apareo bibliográfico (Boyack y Klavans, 2010).

El estudio de los vínculos de citación directa se ha aplicado fundamentalmente para la evaluación de la investigación, con el desarrollo de diversos indicadores estadísticos basados en el recuento del número de citas recibidas por los documentos, como el Factor de Impacto o el Índice 'h'. Así, cuanto mayor es el número de citas que recibe un documento (y por extensión sus autores), mayor es el impacto que ha generado en la comunidad científica, y por tanto, se presupone que el trabajo tiene un mayor mérito, interés y utilidad para el progreso del conocimiento (González-Alcaide, Cas-
tellano-Gómez, Valderrama-Zurián y Aleixandre-Benavent, 2008a). La cuantificación y análisis de los vínculos de citación directa constituye también la medida intuitiva más sencilla para analizar la influencia de los documentos en la literatura posterior, por lo que estos análisis permiten identificar y estudiar las relaciones temáticas existentes entre los documentos.

El análisis de co-citas es un tipo de estudio basado en la identificación y cuantificación de la frecuencia de aparición conjunta de las parejas de documentos recogidos en la bibliografía de las publicaciones científicas estudiadas, lo que permite analizar las interrelaciones establecidas entre los documentos y determinar cuáles son los documentos y autores más influyentes que han sentado las bases intelectuales del área estudiada.

El estudio de los vínculos de citación directa y los análisis de co-citas son metodologías que permiten identificar los documentos de referencia que han fundamentado el desarrollo del conocimiento en una disciplina científica, así como las influencias intelectuales y carácter seminal para la investigación de los documentos y los autores responsables de los mismos (Small, 1973). Ofrecen, en definitiva, una visión "retrospectiva" acerca de cómo ha sido el desarrollo de la investigación, en la medida en que identifican los documentos que han tenido una mayor incidencia en la literatura posterior. Pero para ello, es necesario que haya transcurrido cierto tiempo para que esos documentos puedan haber sido consultados y citados en otros estudios posteriores.

El análisis de los apareos bibliográficos es una metodología basada en la identificación y cuantificación de las referencias bibliográficas comunes citadas por los documentos objeto de estudio, ya que a partir del hecho de que dos documentos compartan al menos una referencia bibliográfica común citada por ambos, se entiende que están temáticamente relacionados y cuanto mayor es el número de referencias bibliográficas compartidas entre dos documentos, mayor es la proximidad temática existente entre los mismos (Kessler, 1963). El interés analítico de los estudios basados en el análisis de los apareos bibliográficos radica en que permite identificar las áreas o núcleos de conocimiento activos existentes dentro de una disciplina. A diferencia de las anteriores, se trata de una metodología "prospectiva", en el sentido de que desde el momento en que se publica o da a conocer un documento (por ejemplo a través de su versión online-first), es posible adscribirlo o agruparlo junto a otros documentos que recogen las mismas referencias bibliográficas y tienen, por tanto, una afinidad temática entre sí, no siendo necesario esperar a que ese documento "circule" entre la comunidad científica y pueda ser consultado y citado; permitiendo además esta metodología conocer el estadio de evolución o desarrollo de un tema (Boyack et al., 2010; Jarneving, 2007a; Jarneving, 2007b). En la figura 1 se ilustran a modo de ejemplo los tres procedimientos descritos de análisis de las referencias bibliográficas de las publicaciones científicas. 


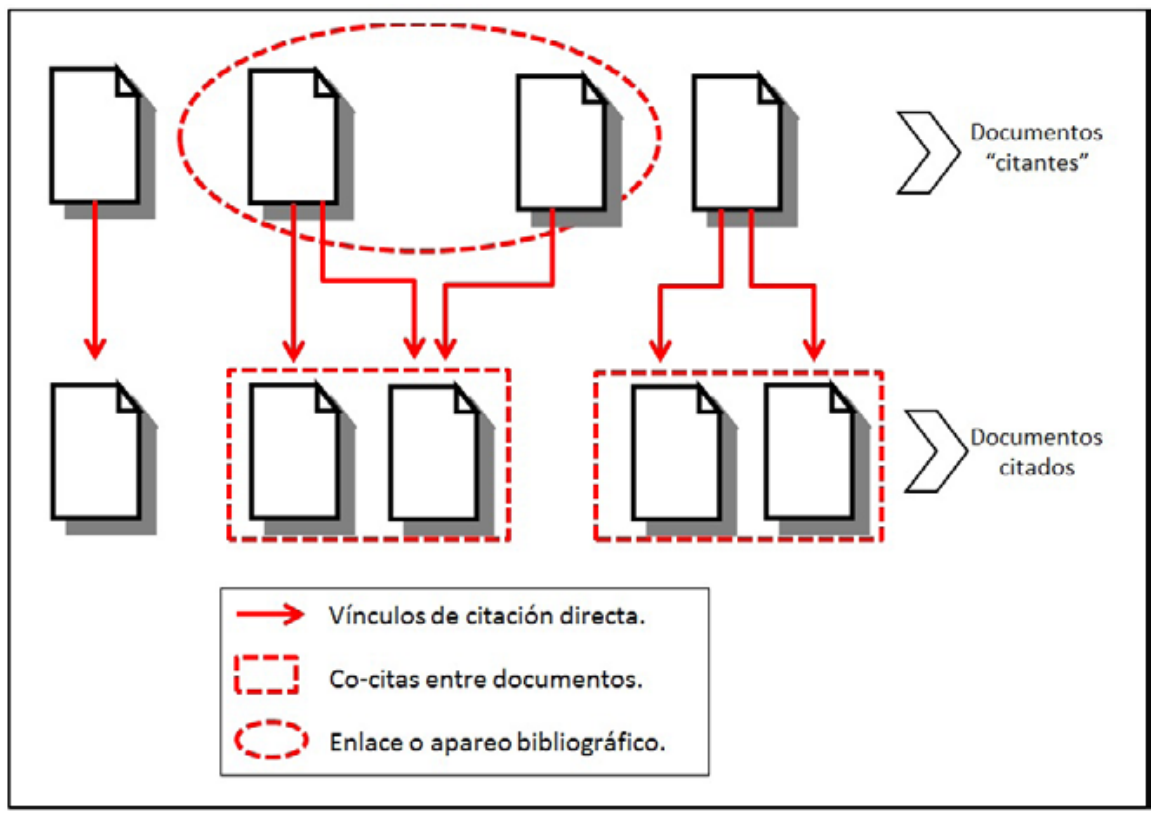

Figura 1. Ejemplos de las metodologías de análisis de las referencias bibliográficas de las publicaciones científicas.

Diferentes estudios han analizado el desarrollo de la investigación española sobre abuso de sustancias a través de indicadores bibliométricos, a nivel nacional o considerando el papel desempeñado por la investigación española en el contexto internacional, si bien, todos los trabajos se han centrado en analizar la producción científica y en menor medida el grado de citación, o más recientemente en el estudio de la colaboración científica en el área (Arbinaga, 2002; De Granda-Orive et al., 2009; González-Alcaide et al., 2006; González-Alcaide et al., 2007; Guardiola, Sánchez-Carbonell, Beranuy y Bellés, 2006; Osca Lluch, 2012; Porcel, Castellano-Gómez, Valderrama-Zurián, Aleixandre-Benavent y Choren, 2003; Sánchez Carbonell, Guardiola, Bellés y Beranuy, 2005). Aunque existen pocos estudios que hayan analizado los ámbitos de investigación de una disciplina o área de conocimiento mediante el análisis de los enlaces bibliográficos en relación con otras metodologías como el análisis de co-citas, esta aproximación metodológica está despertando un interés cada vez mayor, como lo prueba la reciente publicación de numerosas contribuciones que tratan de sentar las bases conceptuales y teóricas de esta metodología (Boyack et al., 2010) así como de diferentes trabajos descriptivos que identifican los ámbitos de investigación existentes en áreas como la Cardiología (Jarneving, 2001), la estrategia empresarial (Vogel y Güttel, 2013), en relación con la literatura generada sobre los diodos orgánicos de emisión de luz (Huang y Chang, 2014) o la investigación sobre turismo (Yuan, Gretzel y Tseng, 2014). También esta metodología se ha utilizado para anticipar avances tecnológicos en el área de la nanotecnología (Kuusi y Meyer, 2007) o para identificar el surgimiento de nuevos ámbitos de investigación (Meyer et al., 2014), entre otros aspectos.
El objetivo del presente estudio consiste en identificar mediante el análisis de las referencias bibliográficas de los documentos, los principales núcleos activos que articulan el desarrollo de la investigación española sobre las adicciones, caracterizando el papel que desempeña cada uno de estos núcleos en el conjunto de la investigación.

\section{Método}

Se ha seleccionado el apareo bibliográfico como la aproximación metodológica más adecuada en relación con el objetivo del estudio, ya que permite identificar los ámbitos temáticos abordados por los investigadores así como establecer el grado o estadio de desarrollo de los mismos. A continuación se describe el proceso seguido a nivel metodológico.

\section{Identificación de la colección de documentos obje- to de estudio}

Para identificar las publicaciones científicas de los investigadores españoles sobre abuso de sustancias y adicciones, se realizó una búsqueda bibliográfica en la Web of Science mediante un perfil de búsqueda que incluía el topónimo "Spain" en el campo "afiliación institucional". La búsqueda fue limitada a los documentos publicados en las revistas recogidas en la categoría Substance Abuse tanto de la edición JCR-Science Edition, como de JCR-Social Sciences Edition, al período cronológico 2000-2013 y a los artículos, revisiones y cartas, con el propósito de centrar el análisis en las principales revistas fuente que conforman el mainstream o corriente principal de la disciplina, en las publicaciones más recientes y en las tipologías documentales que recogen y presentan los resultados originales de la actividad investigadora. 


\section{Organización y tratamiento de la información bibliográfica}

Con la información bibliográfica de los registros recuperados se creó una base de datos relacional, con el propósito de organizar y homogeneizar la información, realizar los cálculos y obtener los indicadores que se describen a continuación.

A cada uno de los documentos recuperados se le asignó una clave numérica para identificarlo de forma unívoca. Asimismo, se procesó y homogeneizó la información recogida en el campo "Cited References" (CR), eliminando por ejemplo los puntos o espacios en blanco tras las iniciales de los nombres de pila y disponiendo únicamente en mayúscula la primera letra de los apellidos de los autores o de cada una de las palabras de los títulos de las revistas.

A continuación se cuantificó para cada una de las parejas de documentos recuperados su frecuencia de apareo bibliográfico, es decir, el número de referencias bibliográficas comunes citadas en ambos documentos, construyendo una matriz de coocurrencia con los valores absolutos de las referencias compartidas por cada pareja de documentos.

Esta matriz de coocurrencia de valores absolutos se transformó en una matriz de correlaciones que recoge el grado de proximidad entre cada pareja de documentos, utilizando para ello como medida de similitud el coeficiente de correlación de Pearson. La transformación de los valores absolutos en una matriz de correlaciones de similitud presenta la ventaja de que cada valor toma en consideración la totalidad de documentos analizados y no únicamente los valores absolutos correspondientes a los pares de documentos en cuestión; asimismo se obtienen valores normalizados, matizándose así las posibles diferencias en la escala de medición entre documentos que comparten un elevado número de referencias comunes y otros documentos que pueden ser también próximos temáticamente aunque con un menor número de referencias compartidas; o entre documentos que presentan una bibliografía mucho más extensa que otros que recogen un menor número de referencias bibliográficas.

\section{Análisis factorial y construcción de la red de enla- ces bibliográficos}

Con la matriz de medidas de similitud se efectuó un análisis factorial para identificar y agrupar los principales documentos que representan los núcleos de la investigación del área. Se identificaron los factores existentes y el número de documentos asignados a cada factor. Se construyó asimismo una red para visualizar la estructura conformada por los núcleos de investigación y analizar las interrelaciones existentes entre los documentos, profundizando en la interpretación de los resultados ofrecidos por el análisis factorial. Con el propósito de centrar el análisis en las publicaciones que presentan un mayor grado de interrelación entre sí e identificar las relaciones principales entre los núcleos de documentos, se ejecutó un algoritmo de "poda" de la red, representando únicamente los pares de documentos con un umbral de coocurrencia de valores absolutos $\geq 4$ y con una medida de similitud $\geq 0.4$.

\section{Caracterización temática de los ámbitos de investi- gación y análisis de resultados}

Cada uno de los factores resultantes del análisis fue etiquetado y descrito. Para ello, se identificaron los principales autores responsables de los documentos agrupados en cada factor y dos expertos analizaron los títulos, palabras clave y resúmenes de los documentos.

Para la representación visual de la red con los núcleos de investigación se utilizó el algoritmo Kamada-Kawai, de uso generalizado para la representación de redes sociales, que distribuye de forma uniforme los nodos en el espacio asignándoles coordenadas tratando de ajustar las distancias reales existentes entre los mismos a distancias teóricas estableciendo distancias uniformes en los enlaces. Los resultados del análisis factorial han sido representados en la red mediante diferentes colores de los nodos, con la identificación de los documentos correspondientes a cada factor.

En relación con los programas empleados para la realización del estudio, para la homogeneización de la información bibliográfica y el para cálculo de la frecuencia de los enlaces bibliográficos se utilizó el gestor de base de datos de Open Office; la generación de las matrices de coocurrencia y de similitud así como el análisis factorial fueron implementados mediante el paquete estadístico R; y finalmente para la representación de la red, se utilizó el programa de análisis y visualización de redes Pajek.

\section{Descripción de la terminología utilizada}

A lo largo del presente estudio se han utilizado los siguientes términos específicos propios de la Bibliometría y el análisis de redes:

Enlace bibliográfico. También referido como apareo bibliográfico o acoplamento bibliográfico (en inglés bibliographic coupling), se trata de una metodología de análisis de la literatura científica para medir las asociaciones existentes entre los documentos propuesta por Michael M. Kessler (1963). Cuando un documento A y otro documento B comparten al menos una referencia bibliográfica común, se dice que entre ellos se ha establecido un enlace bibliográfico. Cuanto mayor es el número de referencias bibliográficas compartidas, mayor es la intensidad o fuerza del enlace bibliográfico y mayor es la proximidad temático-intelectual existe entre esos dos documentos.

Umbral de enlaces bibliográficos. Valor arbitrario fijado con el propósito de eliminar las relaciones triviales y centrar el análisis en las relaciones significativas establecidas entre los documentos. Mediante la aplicación de este umbral se reduce el número de documentos iniciales a un subconjunto o núcleo de documentos que se destacan por su mayor frecuencia de interrelaciones, posibilitando así el análisis y las representaciones visuales (Small, 2009). 
Documentos nucleares. Conjunto de documentos resultante de la aplicación de un umbral 'n' de enlaces bibliográficos y de un umbral ' $r$ ' de similitud a los documentos objeto de estudio (Glänzel y Czerwon, 1996).

Red de enlaces bibliográficos. Representación gráfica de los documentos nucleares. Los nodos representan documentos y los enlaces entre los nodos la existencia de un vínculo de enlace bibliográfico y de una similitud o proximidad temática entre los mismos. Los colores de los nodos identifican las agrupaciones de documentos correspondientes al análisis factorial efectuado.

Dentro de los documentos nucleares es posible distinguir diferentes componentes, siendo cada componente el conjunto de documentos vinculados entre sí de forma directa o a través de intermediarios; y cada componente puede integrar a su vez diferentes clústeres o núcleos de investigación, que son agrupaciones de documentos similares que representan las áreas de conocimiento activas existentes en una disciplina científica y que constituyen entidades homogéneas y cohesionadas diferenciadas de otros clústeres o núcleos de investigación, aunque puedan existir vínculos entre documentos de diferentes clústeres. Existen diferentes metodologías y procedimientos para identificar los clústeres y agrupar los documentos en las redes, habiéndose optado en el presente trabajo por su identificación mediante los resultados del análisis factorial, tal y como se ha realizado en otros estudios previos (Vogel et al., 2013). Los conceptos de factor y de clúster o núcleo de investigación se utilizarán, por tanto, indistintamente en el presente estudio.

Tipo de núcleo de investigación. Determinación del estadio de desarrollo diacrónico de los factores o núcleos de investigación identificados que sirve para explorar o predecir su evolución futura. Siguiendo la clasificación propuesta por Upham y Small (2010), es posible distinguir las siguientes tipologías de núcleos de investigación: emergentes (aquellos que no estaban presentes los años iniciales y que han surgido a lo largo de los últimos años); en expansión (aquellos que tienen una presencia a lo largo de todo el período y que presentan una tendencia creciente en los últimos años); estables (aquellos con una presencia a lo largo de todo el período sin que muestren una tendencia clara al aumento o disminución); decrecientes (aquellos que pese a tener una presencia en los últimos años muestran una tendencia a la reducción en el número de documentos) y extintos (aquellos que han dejado de tener una presencia en los años más recientes).

\section{Resultados}

Se analizaron 818 documentos que incluían 34.242 referencias bibliográficas: 744 artículos $(90,95 \%$ de los documentos) aportaron 29.752 referencias, 52 revisiones $(6,36 \%)$ reunían 4.321 referencias y 22 cartas (2,69\%) 169 referencias. El promedio de referencias bibliográficas por documento considerando todas las tipologías documentales conjuntamente se situó en 41,86, si bien, las revisiones presentaron un promedio mucho más elevado $(83,1)$ frente a los artículos $(39,99)$ y las cartas $(7,68)$. El 82,47\% (n=21.411) de las referencias bibliográficas analizadas fueron citadas una sola vez y el 17,53\% $(\mathrm{n}=4.551)$ fueron citadas en dos o más ocasiones.

En la figura 2 se recoge la evolución diacrónica por año de publicación de los documentos analizados mientras que en la tabla 1 se presenta la distribución de los documentos por revista de publicación. Cabe resaltar el notable incremento en el número de trabajos publicados a partir del año 2008, concentrando los últimos cuatro años casi la mitad del total de la producción científica $(46,94 \%)$ y que Adicciones es la principal revista fuente de publicación de la investigación española en el área, seguida por algunas revistas centradas en el alcoholismo (Alcoholism-Clinical and Experimental Research, Alcohol and Alcoholism y Alcohol) o publicaciones de propósito general en relación con las adicciones (Drug and Alcohol Dependence y Addiction), si bien, también están presentes revistas de muy distinta orientación: centradas en las neurociencias y el estudio de las bases biológicas de las adicciones; en la investigación psicosocial y conductual; en políticas preventivas y educativas en relación con las adicciones; o en el consumo de sustancias como la nicotina o la heroína.

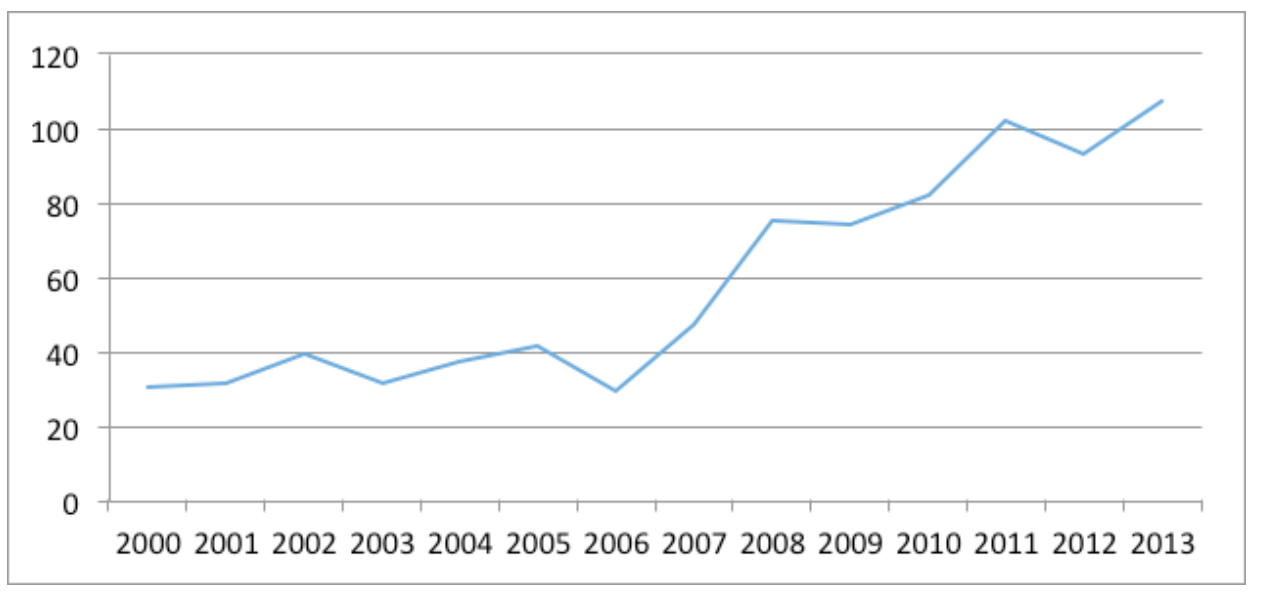

Figura 2. Evolución diacrónica por año de publicación de los documentos analizados 
Tabla 1

Distribución de los documentos analizados por revista de publicación

\begin{tabular}{lcc}
\hline Revista & N docs & $\%$ \\
\hline Adicciones & 160 & 19,56 \\
Alcoholism-Clinical and Experimental Research & 86 & 10,51 \\
Drug and Alcohol Dependence & 79 & 9,66 \\
Alcohol and Alcoholism & 77 & 9,41 \\
Addiction Biology & 53 & 6,48 \\
Addiction & 48 & 5,87 \\
Alcohol & 47 & 5,74 \\
Addictive Behaviors & 41 & 5,01 \\
European Addiction Research & 37 & 4,52 \\
Substance Use \& Misuse & 35 & 4,28 \\
Nicotine \& Tobacco Research & 17 & 2,08 \\
Drug and Alcohol Review & 14 & 1,71 \\
Journal of Substance Abuse Treatment & 12 & 1,47 \\
Heroin Addiction and Related Clinical Problems & 11 & 1,34 \\
Drugs-Education Prevention and Policy & 10 & 1,22 \\
25 revistas con entre 1 y 9 trabajos & 91 & 11,12 \\
\hline Total & 818 & 100 \\
\hline & & \\
\hline
\end{tabular}

Se identificaron 10.391 relaciones de apareo bibliográfico entre parejas de documentos. Un 76,61\% (n=7.545) de esos enlaces bibliográficos únicamente compartieron una referencia bibliográfica en común, un 25,23\% (n=2.622) entre 2 y 9 ; y finalmente un $2,15 \%(n=224)$ de parejas de documentos reunieron más de nueve referencias bibliográficas comunes. El análisis factorial efectuado ha permitido identificar 30 clústeres o núcleos de investigación ( $>4$ documentos). En la tabla 2 se describe la actividad investigadora de cada uno de estos factores, con la indicación de los autores de referencia de los mismos y su estadio de evolución.

La representación de los enlaces bibliográficos entre los documentos en forma de red, ha permitido construir la representación gráfica que se recoge en la figura 3, que constituye una visión de las agrupaciones de documentos nucleares de la investigación española en la disciplina. Se han identificado 446 nodos o documentos destacados vinculados entre sí, entre los que destaca un gran componente que reúne 327 documentos, un segundo componente con
39 documentos y otros cinco componentes que reúnen conjuntamente 46 documentos. Otros 34 documentos que no se recogen en la figura se vinculan entre sí en componentes de menor tamaño (2-4 documentos). Las agrupaciones visuales de carácter intuitivo que se observan a partir del análisis de redes se corresponden en gran medida con los resultados del análisis factorial efectuado. Así, en el conjunto que aglutina el mayor número de documentos vinculados entre sí o componente principal, destacan 21 clústeres de investigación y en el segundo componente se distinguen otros 3 clústeres destacados.

Otros aspectos importantes que cabe señalar en relación con la red construida son la existencia de una mayor densidad de relaciones en algunos clústeres así como la existencia de algunos documentos que se destacan a modo de "puente" o intermediarios que permiten la interconexión entre diferentes clústeres. En la tabla 3 se recogen los 37 documentos más destacados en este sentido, que mantienen vínculos de similitud con varios clústeres de la red. 


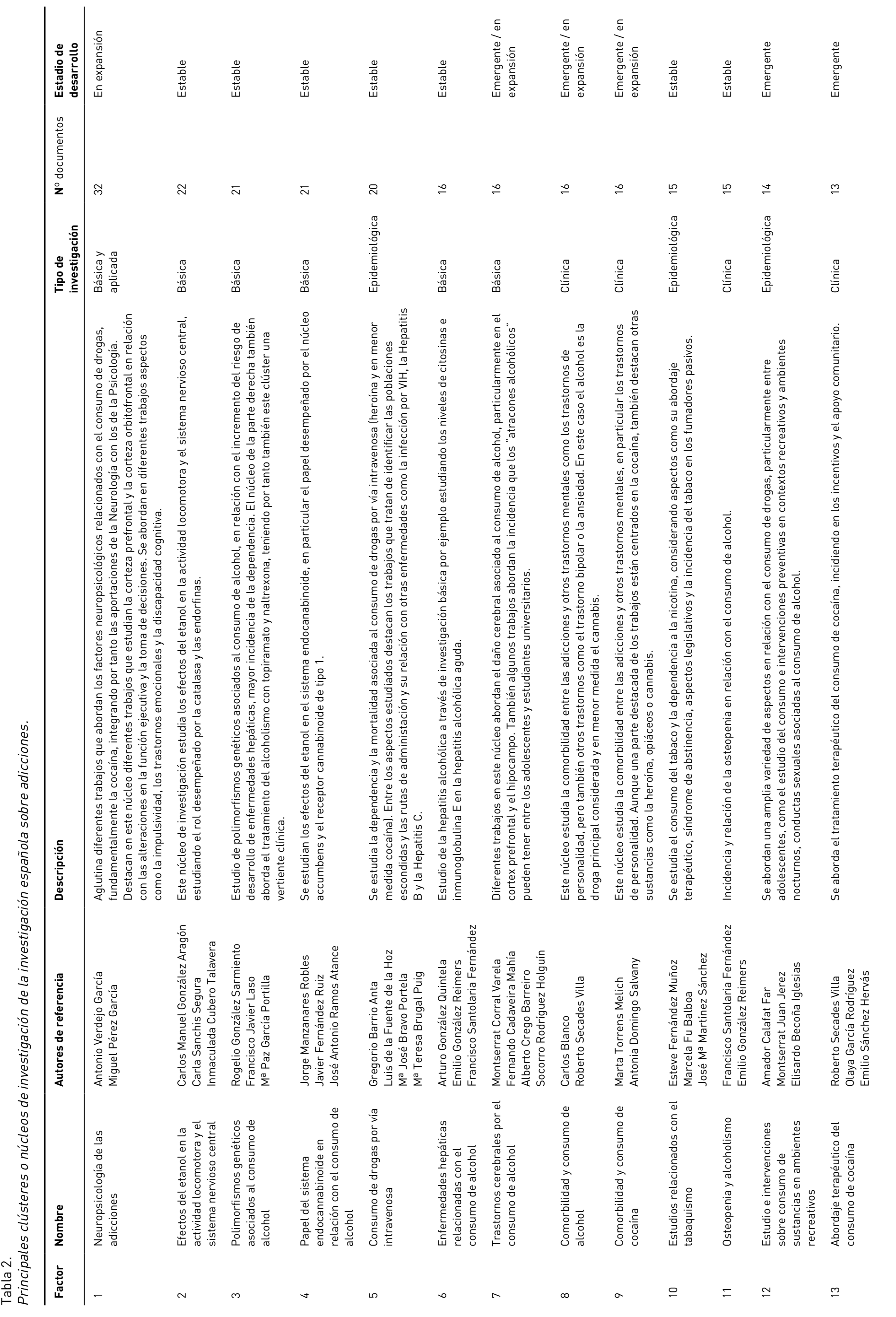




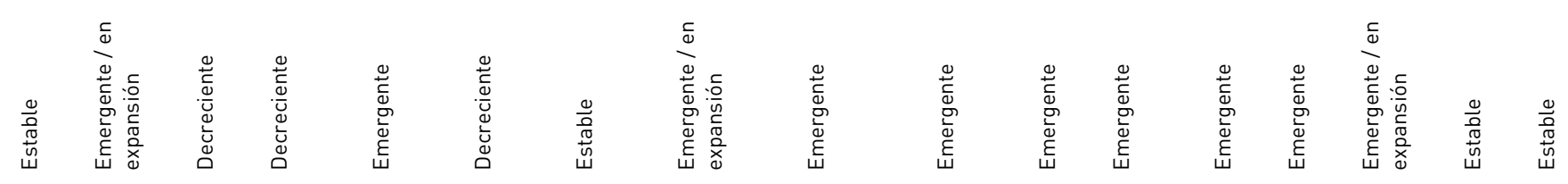

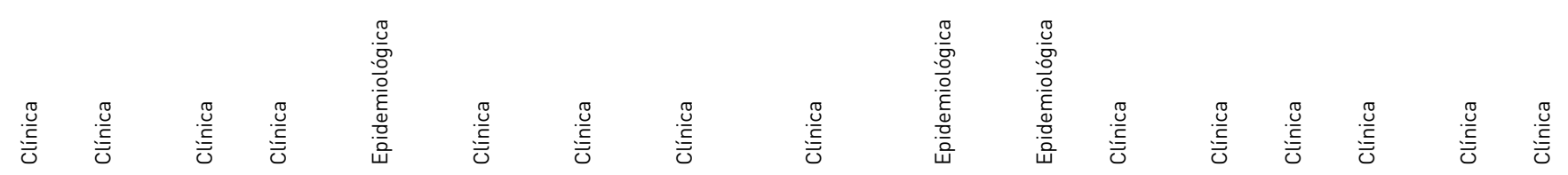

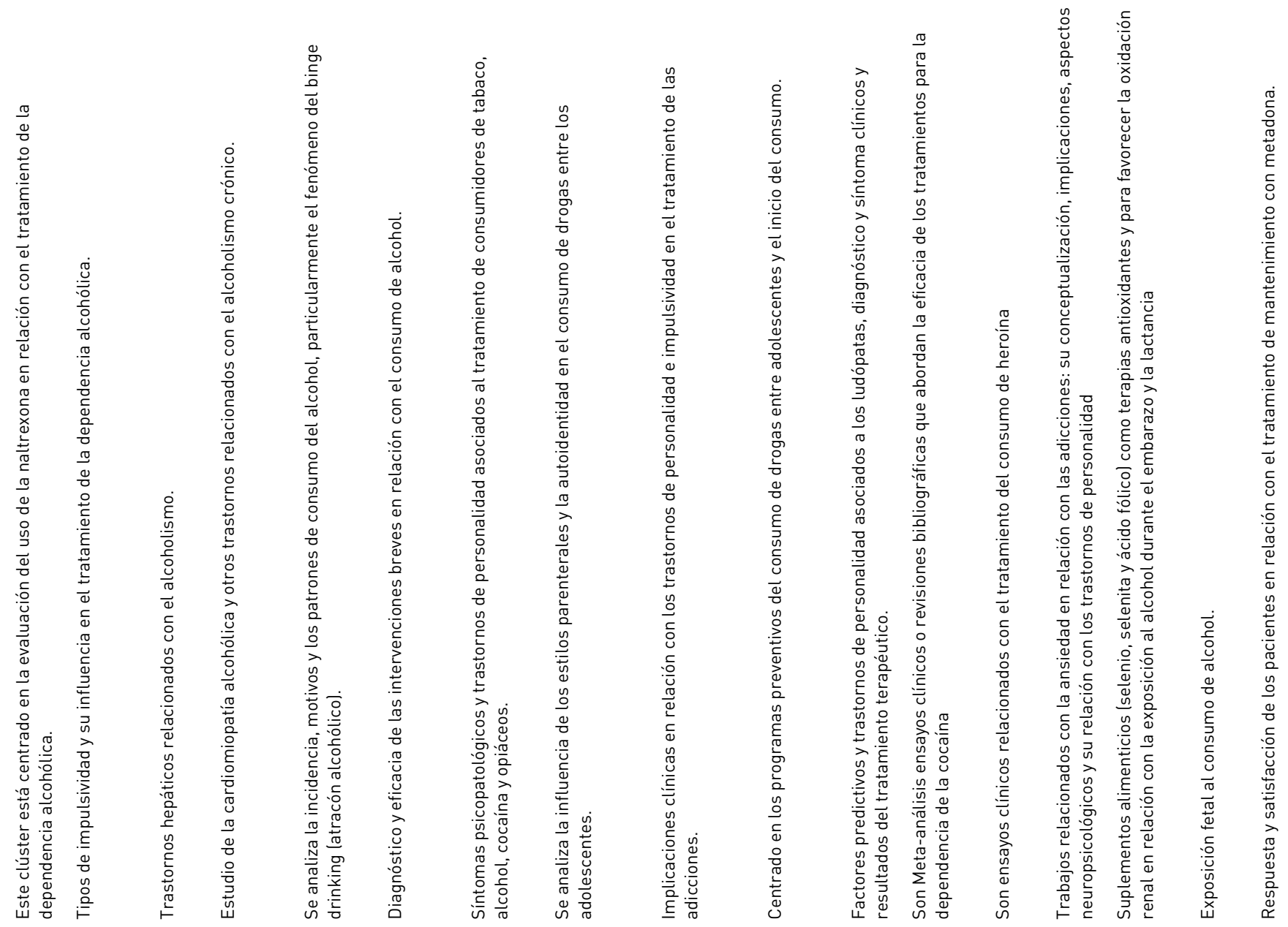

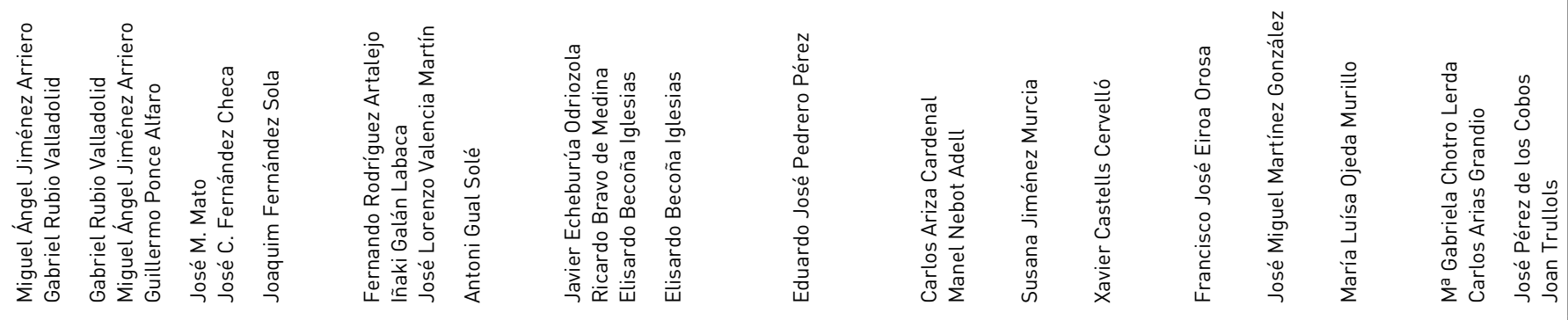

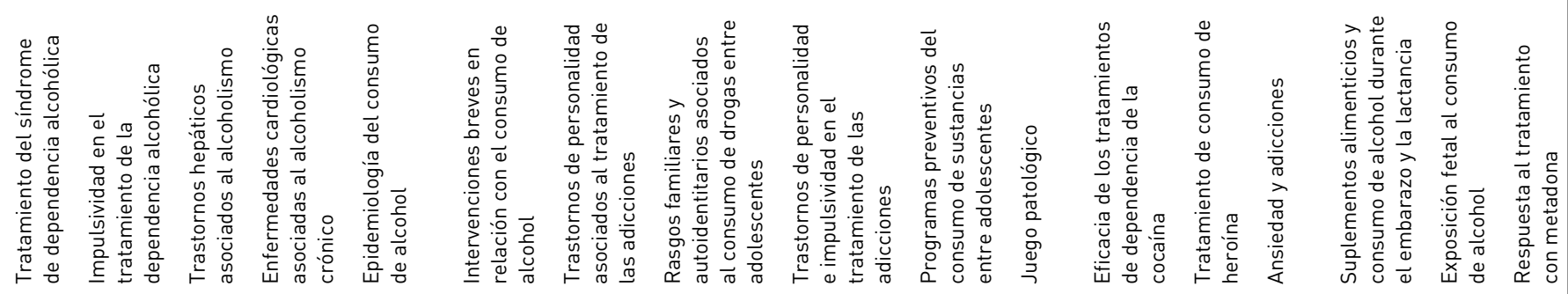

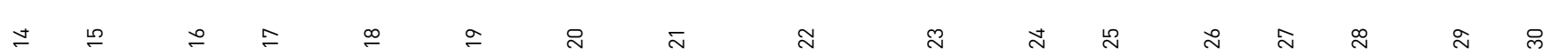


Tabla 3. Documentos más destacados por su intermediación con diferentes clústeres en la investigación española sobre adicciones

\begin{tabular}{|c|c|c|c|}
\hline $\begin{array}{l}N^{0} \text { en } \\
\text { la red }\end{array}$ & Documento & $\begin{array}{c}\text { Factor al que } \\
\text { pertenece }\end{array}$ & $\begin{array}{l}\text { Factores con los } \\
\text { que se relaciona }\end{array}$ \\
\hline
\end{tabular}

1 Marín, M., Ponce, G., Martínez-Gras, I., Koeneke, A., Curivil, P., Jiménez-Arriero, M. A. y Rubio, G. (2012).

15

$3,13,20,24$

Impairments of prepulse inhibition of the startle response in abstinent alcoholic male patients. Alcohol and Alcoholism, 47, 545-551.

2 Marqueta, A., Jiménez-Muro, A., Beamonte, A., Gargallo, P. y Nerín, I. (2010). Evolución de la ansiedad en el proceso de dejar de fumar en fumadores que acuden a una unidad de tabaquismo. Adicciones, 22, 317-324.

3 Walker, M., Toneatto, T., Potenza, M. N., Petry, N., Ladouceur, R., Hodgins, D. C., el-Guebaly, N., Echeburúa, E. y Blaszczynski, A. (2006). A framework for reporting outcomes in problem gambling treatment research: the Banff, Alberta Consensus. Addiction, 101, 504-511.

4 Juárez, J. y Eliana, B. T. (2007). Alcohol consumption is enhanced after naltrexone treatment. AlcoholismClinical and Experimental Research, 31, 260-264.

5 Nieva, G., Bruguera, E., Valero, S. y Casas, M. (2010). Impacto de la sintomatología depresiva en los resultados de un programa de deshabituación tabáquica en el lugar de trabajo. Adicciones, 22, 101-106.

6 Zhou, Y., Colombo, G., Niikura, K., Carai, M. A. M., Femenia, T., García-Gutiérrrez, M. S., Manzanares, J., Ho, A., Gessa, G. L. y Kreek, M. J. (2013). Voluntary Alcohol Drinking Enhances Proopiomelanocortin Gene Expression in Nucleus Accumbens Shell and Hypothalamus of Sardinian Alcohol-Preferring Rats. Alcoholism-Clinical and Experimental Research, 37, Pe131-E140.

7 Benito, A., Haro, G., Orengo, T., González, M., Fornés, T. y Mateu, C. (2012). Dependencia de Opiáceos tipo II o antisocial: utilidad del Modelo Psicobiológico de Cloninger en adicciones. Adicciones, 24, 131-138.

8 Vergara-Moragues, E., González-Sáiz, F., Lozano, O. M. y Verdejo García, A. (2013). Psychopathological stability of personality disorders in substance abuse patients treated in a therapeutic community. Journal of Addictive Diseases, 32, 343-353.

9 Nocón, A., Berge, D., Astals, M., Martín-Santos, R. y Torrens, M. (2007). Dual diagnosis in an inpatient drug-abuse detoxification unit. European Addiction Research, 13, 192-200.

10 Marcos, M., Pastor, I., González-Sarmiento, R. y Laso, F. J. (2009). A functional polymorphism of the NFKB1 gene increases the risk for alcoholic liver cirrhosis in patients with alcohol dependence. Alcoholism-Clinical and Experimental Research, 33, 1.857-1.862.

11 Verdura Vizcaíno, E. J., Fernández-Navarro, P., Blanco, C., Ponce, G., Navio, M., Moratti, S. y Rubio, G. (2013). Maintenance of attention and pathological gambling. Psychology of Addictive Behaviors, 27, 861867.

12 Jiménez-Murcia, S., Álvarez-Moya, E. M., Stinchfield, R., Fernández-Aranda, F., Granero, R., Aymamí, N., Gómez-Peña, M., Jaurrieta, N., Bove, F. y Menchón, J. M. (2010). Age of onset in pathological gambling: clinical, therapeutic and personality correlates. Journal of Gambling Studies, 26, 235-248.

13 Del Río, M. C., González-Luque, J. C. y Álvarez, F. J. (2001). Alcohol-related problems and fitness to drive. Alcohol and Alcoholism, 36, 256-261.

14 Jiménez-Murcia, S., Stinchfield, R., Álvarez-Moya, E., Jaurrieta, N., Bueno, B., Granero, R., Aymamí, M. N., Gómez-Peña, M., Martínez-Giménez, R., Fernández-Aranda, F. y Vallejo, J. (2009). Reliability, validity, and classification accuracy of a Spanish translation of a measure of DSM-IV diagnostic criteria for pathological gambling. Journal of Gambling Studies, 25, 93-104

15 Echeburúa, E., González-Ortega, I., de Corral, P. y Polo-López, R. (2011). Clinical gender differences among adult pathological gamblers seeking treatment. Journal of Gambling Studies, 27, 215-227.

16 Adan, A. (2012). Impulsividad funcional y disfuncional en jóvenes con consumo intensivo de alcohol (binge drinking). Adicciones, 24, 17-22.

17 Del Río, E. F., Becoña, E. y Durán, A. L. (2011). Subtypes of smokers who attend psychological treatment in order to stop smoking. Substance Use \& Misuse, 46, 1.113-1.123.

18 Vázquez, M. L., Castillo, I. I., Jiménez-Lerma, J. M., Hormaechea Beldarrain, J. A. y Gutiérrez-Fraile, M. (2009). Clinical trial on the use of olanzapine in reducing the consumption of cocaine in methadone maintenance programmes. Heroin Addiction and Related Clinical Problems, 11, 21-29.

19 Rafful, C., García-Rodríguez, O., Wang, S., Secades-Villa, R., Martínez-Ortega, J. M. y Blanco, C. (2013). Predictors of quit attempts and successful quit attempts in a nationally representative sample of smokers. Addictive Behaviors, 38, 1.920-1.923

20 Ochoa, C., Álvarez-Moya, E. M., Penelo, E., Aymamí, M. N., Gómez-Peña, M., Fernández-Aranda, F., Granero, R., Vallejo-Ruiloba, J., Menchón, J. M., Lawrence, N. S. y Jiménez-Murcia, S. (2013). Decisionmaking deficits in pathological gambling: The role of executive functions, explicit knowledge and impulsivity in relation to decisions made under ambiguity and risk. American Journal on Addictions, 22, 492-499.

21 Pedrero Pérez, E. J. y Rojo Mota, G. (2008). Diferencias de personalidad entre adictos a sustancias y población general. Estudio con el TCl-R de casos clínicos con controles emparejados. Adicciones, 20, 251 261. 
23 Femenía, T. y Manzanares, J. (2012). Increased ethanol intake in prodynorphin knockout mice is associated to changes in opioid receptor function and dopamine transmission. Addiction Biology, 17, 322-337.

24 Cuenca-Royo, A. M., Sánchez-Niubó, A., Forero, C. G., Torrens, M., Suelves, J. M. y Domingo-Salvany, A. (2012). Psychometric properties of the CAST and SDS scales in young adult cannabis users. Addictive Behaviors, 37, 709-715.

25 McCormick, R., Docherty, B., Segura, L., Colom, J., Gual, A., Cassidy, P., Kaner, E. y Heather, N. (2010). The research translation problem: Alcohol screening and brief intervention in primary care - Real world evidence supports theory. Drugs-Education Prevention and Policy, 17, 732-748.

26 González-Sáiz, F., Domingo-Salvany, A., Barrio, G., Sánchez-Niubó, A., Brugal, M. T., de la Fuente, L. y Alonso, J. (2009). Severity of Dependence Scale as a Diagnostic Tool for Heroin and Cocaine Dependence. European Addiction Research, 15, 87-93.

27 Verdejo-García, A., Bechara, A., Recknor, E. C. y Pérez-García, M. (2007). Negative emotion-driven impulsivity predicts substance dependence problems. Drug and Alcohol Dependence,91, 213-219.

28 Anderson, P. (2009). Overview of interventions to enhance primary-care provider management of patients with substance-use disorders. Drug and Alcohol Review, 28, 567-574.

29 Nieva, G., Ortega, L. L., Mondón, S., Ballbè, M. y Gual, A. (2011). Simultaneous versus delayed treatment of tobacco dependence in alcohol-dependent outpatients. European Addiction Research, 17, 1-9.

30 Moreno, M., Estévez, A. F., Zaldívar, F., García Montes, J. M., Gutiérrez-Ferre, V. E., Esteban, L., SánchezSanted, F. y Flores, P. (2012). Impulsivity differences in recreational cannabis users and binge drinkers in a university population. Drug and Alcohol Dependence, 124, 355-362.

31 Colom Farran, J., Casas, M., Pérez de los Cobos, J., del Río, M., Roncero, C., Castells, X., Valero, S. Eiroa-Orosa, F. J., Batlle, F. y Trujols, J. (2012). Feasibility of double-blind clinical trials with oral diacetylmorphine: a randomized controlled phase II study in an inpatient setting. European Addiction Research, 18, 279-287.

32 Valencia-Martín, J. L., Galán, I. y Rodríguez-Artalejo, F. (2009). Alcohol and self-rated health in a mediterranean country: the role of average volume, drinking pattern, and alcohol dependence. Alcoholism-Clinical and Experimental Research, 33, 240-246.

33 Sánchez-Hervás, E., Zacarés Romaguera, F., Santonja Gómez, F. J., Secades-Villa, R., García-Rodríguez, O. y Martín Yáñez, E. (2010). Urine testing during treatment predicts cocaine abstinence. Journal of Psychoactive Drugs, 42, 347-352.

34 Sánchez-Hervás, E. y Llorente del Pozo, J. M. (2012). Recaídas en la adicción a cocaína: una revisión. Adicciones, 24, 269-279.

35 Santolaria, F., González-Reimers, E., Pérez-Manzano, J. L., Milena, A., Gómez-Rodríguez, M. A., González Díaz, A., de la Vega, M. J. y Martínez-Riera, A. (2000). Osteopenia assessed by body composition analysis is related to malnutrition in alcoholic patients. Alcohol, 22, 147-157.

36 González-Reimers, E., Fernández-Rodríguez, C. M., Santolaria-Fernández, F., de la Vega-Prieto, M. J., Martín-González, C., Gómez-Rodríguez, M. A., Alemán-Valls, R. y Rodríguez-Gaspar, M. (2011). Interleukin-15 and other myokines in chronic alcoholics. Alcohol and Alcoholism, 46, 529-533. 


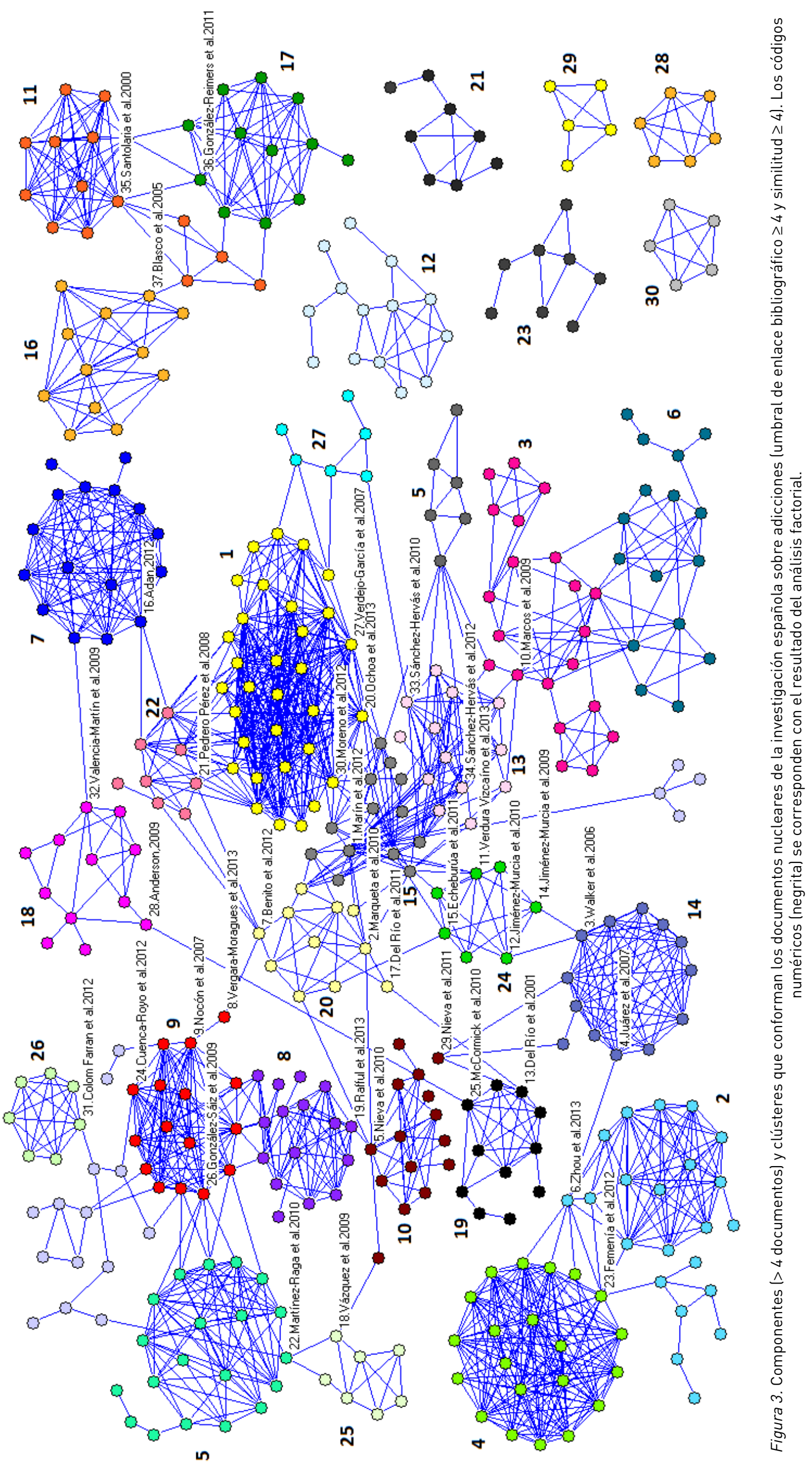




\section{Discusión}

La mayor parte de los estudios que miden el desarrollo de las actividades científicas a través de indicadores bibliométricos se han centrado en la evaluación de la investigación, particularmente mediante el uso de indicadores de producción o de impacto. Sin embargo, la información ofrecida por aproximaciones como la del presente estudio, puede resultar de mayor interés para los organismos administradores y financiadores de cara a facilitar la toma de decisiones acerca de los planes de actuación a desarrollar en relación con la investigación, ya que el análisis de los enlaces bibliográficos es una metodología que permite identificar las áreas más activas de la investigación y evaluar la competitividad nacional, reforzando los ámbitos clave de desarrollo científico. También resulta de utilidad para identificar los temas emergentes e innovadores, anticipar avances tecnológicos o en la investigación y determinar qué paradigmas dominarán la investigación en el futuro (Boyack y Klavans, 2014; Huang et al., 2014; Kuusi et al., 2007). En relación con los investigadores y profesionales del área, la información derivada del análisis de los enlaces bibliográficos puede resultar de utilidad para conocer cuáles son las áreas de conocimiento y los conceptos clave de la disciplina y la posición que ocupan en relación con los mismos o para identificar oportunidades de investigación (Yuan et al., 2014).

El análisis factorial ha mostrado unos resultados consistentes, ya que los expertos consultados confirman que los núcleos de investigación identificados representan las principales áreas activas de investigación de la disciplina, permitiendo incluso distinguir diferentes núcleos en los que pese a abordarse un mismo aspecto, como la comorbilidad o patología dual (en el caso de los clústeres 8 y 9), ésta es estudiada desde diferentes ópticas: en el primero de los casos se estudian los trastornos de personalidad asociados al consumo de alcohol desde una óptica epidemiológica; y en el segundo caso se abordan los trastornos de personalidad asociados a la adicción a la cocaína mediante el empleo de las entrevistas como metodología de investigación.

Siguiendo una distinción tradicional de los tipos de investigación científica en el área biomédica, en relación con los núcleos o clústeres de la investigación española del área del abuso de sustancias, nos encontramos, por una parte, con diferentes clústeres o núcleos de investigación básica, que tratan de ampliar el conocimiento acerca del fenómeno estudiado a través de la investigación en laboratorios y, por otra parte, otros núcleos de investigación aplicada. Entre estos últimos, algunos núcleos que pueden ser definidos como clínicos y psicosociales, en tanto que presentan los resultados del abordaje terapéutico de las adicciones o el tratamiento de las enfermedades asociadas a las mismas; o bien tratarse de núcleos de investigación epidemiológicos, de gran importancia en relación con las adicciones para fundamentar los factores de riesgo y las intervenciones preventivas, entre otros aspectos. Una distribución similar de núcleos de investigación, básica y clínica, ha sido observada en el área cardiológica (Jarneving, 2001). Cabe destacar en relación con esta agrupación que los clústeres presentan características diferentes: los núcleos de investigación básica aglutinan a un mayor número de documentos y presentan una mayor densidad de relaciones en relación con los núcleos de investigación aplicada, lo que puede responder a diferentes motivos, como la vinculación de la investigación básica a universidades y centros de investigación y a grupos más numerosos donde existe una mayor presión por publicar, frente a la investigación clínica, más atomizada al estar vinculada a centros asistenciales y a disciplinas de áreas psicosociales, con un menor peso de las prácticas cooperativas (González Alcaide et al., 2006).

A nivel de sustancias, domina el alcohol, presente en 17 de los núcleos de investigación, si bien, también la cocaína tiene una destacada presencia en 6 núcleos. La heroína y los opiáceos únicamente están presentes como droga destacada en 4 núcleos y el cannabis y el tabaco en otros dos cada uno de ellos, respectivamente. Los núcleos que estudian la relación entre las adiciones y aspectos como los trastornos de personalidad, la ansiedad o la implementación de programas preventivos abordan el estudio de varias sustancias. La escasa presencia del tabaco, uno de los sectores más activos en la investigación a nivel mundial, se puede explicar por el hecho de que una parte destacada de la investigación no está centrada en la investigación desde la óptica de las adicciones, sino en relación con las enfermedades asociadas al tabaquismo, con un mayor peso de disciplinas como la Oncología o el Sistema Respiratorio o en aspectos de Salud Pública tal y como se ha mostrado en estudios previos (García López, 1999). Este mismo motivo puede haber conllevado el hecho de que no estén presentes otros núcleos, particularmente de la investigación básica de áreas como la Gastroenterología y Hepatología o la Cardiología que sí que están presentes dentro de las líneas de la Red de Trastornos Adictivos constituida en el área. También resulta llamativa la escasa presencia del juego patológico, presente únicamente en un pequeño núcleo, no habiéndose detectado ningún núcleo activo en relación con otras adicciones de gran actualidad que podrían constituirse en destacados temas objeto de atención por parte de la investigación, como las adicciones a los teléfonos móviles, a Internet o a las nuevas tecnologías (Echeburúa y Corral, 2010).

Los trabajos identificados en el presente estudio a través del análisis de los apareos bibliográficos constituyen los documentos nucleares de la disciplina analizada, siguiendo el concepto introducido por Glänzel y Czerwon (1995). Y las agrupaciones que conforman estos documentos representan los temas candentes o más destacados de la investigación. Algunas de las implicaciones más interesantes de su estudio son dos destacadas propiedades asociadas a este núcleo de documentos: por una parte tienden a concentrar con el tiempo un elevado grado de citación (Jarneving, 
2013); y por otra parte, suelen asociarse con otros nuevos documentos que se van publicando en el área (Glänzel et al., 1996). Por lo tanto, esta metodología además de ofrecer una panorámica de los temas que se abordan en la investigación actual, constituiría un método de detección temprana de los documentos más destacados que probablemente se erigirán en los trabajos de referencia de la disciplina (Jarneving, 2007b). Asimismo, esta metodología permite determinar el estadio de evolución de los núcleos de investigación identificados y la detección de ámbitos o temas emergentes (Glänzel y Thijs, 2012; Upham et al., 2010). En lo referente al estadio de desarrollo de los ámbitos de investigación identificados en el presente estudio, el predominio de los núcleos de investigación emergentes o en expansión (15 de los núcleos identificados), aun considerando la posible incidencia que ha podido tener la inclusión de la revista Adicciones en la base de datos estudiada el año 2008, cabe interpretarlo como un rasgo positivo y reflejo, en cualquier caso, del proceso de internacionalización de la investigación española y de la progresiva articulación y consolidación de una comunidad científica en la disciplina (González-Alcaide, Valderrama-Zurián y Aleixandre-Benavent, 2012). Resultaría fundamental dar continuidad a los grupos y las líneas de investigación incipientes o en expansión identificadas, con una adecuada planificación de la Ciencia que dé respuesta a las "verdaderas" necesidades de España en un área tan sensible como las adicciones y no dejar que la investigación se desarrolle únicamente a partir del interés e iniciativa individual de los investigadores y grupos de investigación.

La representación de los núcleos de investigación en forma de red, ha permitido complementar el análisis factorial efectuado, analizando las agrupaciones de los documentos y los vínculos establecidos entre los diferentes núcleos de investigación. La imagen global de la estructura de la red, ofrece una panorámica de una investigación dispersa con numerosos núcleos diferenciados con pocas conexiones entre sí, lo que refiere cierto grado de atomización de la investigación. La distribución de los núcleos de investigación entre diferentes dominios de conocimiento propios de la disciplina (por ejemplo en función del tipo de sustancia estudiada o la orientación de la investigación, básica o aplicada) puede explicar en parte la dispersión de los núcleos de investigación, tal y como se ha observado en estudios similares realizados en otras áreas de conocimiento (Huang et al., 2014), en contraste con lo que sucede en otras áreas que muestran, en cambio, una elevada concentración de documentos en torno a un único núcleo o tema de investigación (Yuan et al., 2014). No obstante, el hecho de que existan diferentes núcleos que pese a abordar una misma temática o problema de investigación (como los núcleos 20 y 22), no se presenten conectados entre sí o tengan vínculos puntuales, cabe interpretarlo en clave de una falta de "consenso científico”, ya que esa falta de conexión está reflejando líneas de investigación fundamentadas en bases intelectuales di- ferentes y probablemente con liderazgos diferentes dentro de cada núcleo o grupo, siendo el análisis de los enlaces bibliográficos una metodología de investigación de gran utilidad para detectar este rasgo de la investigación (Nicolaisen y Frandsen, 2012).

Solamente unos pocos autores aparecen como investigadores destacados en varios núcleos (Elisardo Becoña Iglesias, Emilio González Reimers, Miguel Ángel Jiménez Arriero, Gabriel Rubio Valladolid, Francisco Santolaria Fernández y Roberto Secades Villa), lo que refuerza la idea de la atomización de la investigación en el área y la necesidad de potenciar la cohesión de la misma, ya que la destacada presencia de investigadores consolidados de referencia constituye uno de los principales indicadores de madurez de una disciplina científica. La falta de conciencia acerca del hecho de que se trabaja dentro de una comunidad científica puede contribuir a la atomización y suponer una fragilidad para el desarrollo de la disciplina. El refuerzo de los vínculos comunitarios y cooperativos, incidiendo en los beneficios que se pueden derivar de la colaboración científica, particularmente de determinadas prácticas cooperativas, como los vínculos de colaboración internacional, interdisciplinares o entre diferentes sectores o tipos de investigación, puede ser uno de los mecanismos para favorecer la cohesión del área.

Aunque en general hay poca conexión entre los núcleos de investigación identificados, es posible establecer una diferenciación entre ellos. Así, en el centro de la red del componente principal se sitúan diferentes núcleos de investigación que abordan el tratamiento clínico o terapéutico del consumo de drogas (como los núcleos 10, 13, 14, 15, 19 y 24), presentando una mayor interconexión entre sí y con otros núcleos que los núcleos de investigación básica, que se presentan mayoritariamente en la periferia de la red, con una destacada cohesión interna derivada de la elevada "densidad" de interrelaciones que mantienen los documentos que los integran entre sí, pero con un marcado "aislamiento" con menores vínculos externos con el resto de núcleos. Esa mayor centralidad de la práctica clínica, al igual que en el caso del conocimiento aplicado y la transferencia tecnológica, puede responder al hecho de que estos aspectos constituyen el fin último de la investigación, integrando el conocimiento aportado desde diferentes aproximaciones y líneas de investigación. El hecho de que los núcleos de investigación básica presenten un carácter "introspectivo" y en gran medida "aislado" en la periferia de la red, cerrados en sí mismos y siendo poco permeables a la influencia de otros ámbitos del conocimiento, aunque puede ser un rasgo propio de la investigación básica, si se presenta muy acentuado puede resultar negativo, ya que las influencias externas pueden constituirse en un destacado mecanismo para favorecer la innovación y el avance del conocimiento (Long, Cunningham y Braithwaite, 2012). Los núcleos que tratan enfermedades, aspectos epidemiológicos y con carác- 
ter general la investigación psicosocial (como los núcleos 3,6 y 18), se presentan también en la periferia de la red, con una menor densidad de vínculos internos, pero con un mayor grado de apertura y conexiones con otros núcleos. El análisis de redes analizando la centralidad de los núcleos de investigación puede constituirse en una herramienta metodológica útil para caracterizar el grado de conectividad y las interrelaciones de la investigación en una disciplina o área de conocimiento, evaluando cuál es la forma óptima de generación de nuevo conocimiento (Youtie, Kay y Melkers, 2013).

El análisis y la representación de los núcleos de investigación en forma de red, permite también la identificación y un análisis muy preciso de los documentos que desempeñan un papel clave para asegurar la conexión de unos núcleos con otros, al funcionar a modo de intermediarios entre diferentes clústeres, lo que cabe interpretar en clave de que estos documentos tienen un particular valor al favorecer la conectividad y cohesión de la investigación en la disciplina (Meyer et al., 2014). Se ha observado que este destacado papel de intermediarios responde en muchos casos al hecho de que se trata de documentos que abordan problemáticas transversales comunes a varios ámbitos de investigación o bien que se trata de tipologías documentales como ensayos clínicos o revisiones, documentos de propósito general con un alto nivel de evidencia que sitúan el conocimiento existente sobre el tema abordado, y por tanto, son más proclives a ser considerados documentos de referencia y a ser citados en estudios posteriores. Al igual que en el caso de los autores, sería deseable la existencia de un número más destacado de trabajos de referencia citados por diferentes clústeres que fijasen el consenso científico existente sobre el tema estudiado, lo que constituiría un destacado indicador acerca de la cohesión y madurez de la disciplina.

Como limitaciones del estudio realizado cabe apuntar todas aquellas inherentes a los estudios bibliométricos basados en los análisis de citas, entre las que se pueden señalar sesgos derivados de la posible existencia de errores en las referencias bibliográficas, que no se tiene presente en el análisis el sentido o propósito de las citas o la posible incidencia de las autocitas (MacRoberts y MacRoberts, 1996). En relación con el uso del enlace bibliográfico como método de investigación, hay que apuntar que no se evalúa el impacto o visibilidad de los trabajos, a diferencia de otros métodos como los análisis de citación directa o de co-citas (Jarneving, 2005). También es importante señalar que se han analizado únicamente los documentos publicados en las revistas especializadas en adicciones, cuando se trata de un área de conocimiento con un marcado carácter multidisciplinar, y por tanto, con una importante dispersión de las publicaciones en otras áreas de conocimiento, tanto entre áreas biomédicas como de Salud Pública o Psicología, entre otras (González-Alcaide et al., 2008b). Se ha optado por este enfoque para centrar el estudio en la corriente principal de la disciplina, que es la liderada por las publicaciones especializadas del área, evitando así que aspectos como un interés marginal por el estudio de las adicciones generen "ruido documental" (o recuperación de documentos no relevantes); que núcleos de investigación, aunque relacionados con el abuso de sustancias, más vinculados a otras especialidades médicas como la Neumología o la Gastroenterología y Hepatología; o que líneas de investigación transversales que no representen las problemáticas y el objeto de estudio central de la disciplina distorsionen los resultados del estudio (Arciniega y Miller, 1997).

En relación con la cobertura de revistas de las bases de datos de Thomson Reuters y los posibles sesgos derivados de la misma, hay que señalar que el método de investigación aplicado no es especialmente sensible a este problema, ya que no se quedan fuera del análisis documentos de referencia, sino que se clasifican y agrupan los documentos identificados, siendo difícil que autores y ámbitos temáticos consolidados tengan únicamente una proyección nacional o no hayan sido identificados, máxime teniendo presente que los criterios de calidad de los organismos responsables de las políticas científicas en España toman como referencia para la práctica totalidad de procesos evaluativos las revistas incluidas en la cobertura de las bases de datos analizadas, y por tanto, los autores tratan de publicar sus trabajos en estas revistas de referencia (Calafat, 2006).

El presente estudio ha permitido identificar las áreas de investigación más destacadas existentes en la investigación española sobre abuso de sustancias y adicciones, mediante un análisis de los enlaces bibliográficos, metodología basada en la cuantificación de la bibliografía común citada en los documentos publicados en las principales revistas del área y en el establecimiento de agrupaciones entre los documentos identificados a partir de su similitud o proximidad temática. Las agrupaciones resultantes de este tipo de análisis han sido definidas a nivel bibliográfico como clústeres o núcleos de investigación, es decir, ámbitos que conforman la vanguardia del conocimiento y que permiten conocer las tendencias principales de investigación, actuales y futuras, en una disciplina o área de conocimiento.

Se ha constatado la existencia de un importante grado de atomización en el área, con la existencia de numerosos núcleos de investigación pero con pocas conexiones entre sí, con un reducido número de documentos que recogen un conocimiento común compartido. Destaca también el elevado número de núcleos emergentes, reflejo de un estado incipiente de muchos de los temas de investigación. Se debe incidir en prácticas que fomenten el consenso científico y la cohesión de la disciplina, así como favorecer la consolidación de las principales líneas que den respuesta a los problemas sociales y desafíos de la investigación.

Posibles líneas de trabajo futuro pueden profundizar en esta línea de investigación, identificando a través de un análisis de co-citas cuáles son los trabajos y autores de referen- 
cia que han sentado las bases conceptuales y la estructura intelectual de la investigación en relación con los diferentes temas o analizando cuál es el impacto de la investigación española en la literatura internacional del área. También ampliar la cobertura geográfica del estudio, analizando el desarrollo de la investigación en otros países o a nivel mundial, ofrecería referentes comparativos para determinar si se siguen los mismos patrones de distribución de la investigación, o acerca de la existencia de "especializaciones" o áreas desatendidas por parte de la investigación.

\section{Bibliografía}

Arbinaga, F. (2002). Análisis bibliométrico de la revista Adicciones (1996-2000). Adicciones, 14, 139-144.

Arciniega, L. T. y Miller, W. R. (1997). Where to publish? Some considerations among English-language addiction journals. Addiction, 92: 1639-1648. doi:10.1111/j.1360-0443.1997.tb02883.x.

Boyack, K. W. y Klavans, R. (2010) . Co-citation analysis, bibliographic coupling, and direct citation: which citation approach represents the research front most accurately? Journal of the American Society for Information Science and Technology, 61, 2389-2404. doi: 10.1002/asi.21419.

Boyack, K. W. y Klavans, R. (2014). Creation of a highly detailed, dynamic, global model and map of Science. Journal of the Association for Information Science and Technology, 65, 670-685. doi: 10.1002/asi.22990.

Calafat, A. (2006). The invisible college of non-English addiction journals. Addiction, 101, 638-645. doi: 10.1111/j.1360-0443.2006.01466.x.

Culebras-Fernández, J., García de Lorenzo, A., Wanden-Nerghe, C., David Castiel, L. y Sanz-Valero, J. (2008). ¡Cuidado!, sus referencias bibliográficas pueden ser estudiadas. Nutrición Hospitalaria, 23, 85-88.

De Granda-Orive, J. I., Villanueva Serrano, S., Aleixandre-Benavent, R., Valderrama-Zurián, J. C., Alonso-Arroyo, A., García-Río, F.,... González-Alcaide, G. (2009). Redes de colaboración científica internacional en tabaquismo: análisis de coautorías mediante el Science Citation Index durante el periodo 1999-2003. Gaceta Sanitaria, 23, 222e34-43.

Echeburúa, E. y Corral, P. (2010). Adicción a las nuevas tecnologías y a las redes sociales en jóvenes: un nuevo reto. Adicciones, 22, 91-96.

García López, J. A. (1999). Bibliometric analysis of Spanish scientific publications on tobacco use during the period 1970-1996. European Journal of Epidemiology, 15, 23-28.

Garfield, E. (1955). Citation Indexes for Science: a new dimension in Documentation through association of ideas. Science, 122, 108-111. doi: 10.1093/ije/dyl189.

Glänzel, W. y Czerwon, H. J. (1995). A new methodological approach to bibliographic coupling and its application to research-front and other core documents. En: Pro- ceedings of $5^{\text {th }}$ International Conference on Scientometrics and Informetrics (pp. 167-176). Chicago: ISSI.

Glänzel, W. y Czerwon, H. J. (1996). A new methodological approach to bibliographic coupling and its application to the national, regional and institutional level. Scientometrics, 37, 195-221. doi: 10.1007/BF02093621.

Glänzel, W. y Thijs, B. (2012). Using 'core documents' for detecting and labeling new emerging topics. Scientometrics, 91, 399-416. doi: 10.1007/s11192-011-0591-7.

González-Alcaide, G., Valderrama-Zurián, J. C., Aleixandre-Benavent, R., Alonso-Arroyo, A., De Granda, J. I. y Villanueva Serrano, S. (2006). Redes de coautoría y colaboración de las instituciones españolas en la producción científica sobre drogodependencias en biomedicina 1999-2004. Trastornos Adictivos, 8, 78-114.

González-Alcaide, G., Valderrama-Zurián, J. C., Navarro Molina, C., Alonso-Arroyo, A., Bolaños Pizarro, M. y Aleixandre Benavent, R. (2007). Análisis de género de la producción científica española sobre drogodependencias en biomedicina 1999-2004. Adicciones, 19, 45-50.

González-Alcaide, G., Castellano-Gómez, M., Valderrama-Zurián, J. C. y Aleixandre-Benavent, R. (2008a). Literatura científica de autores españoles sobre análisis de citas y Factor de Impacto en Biomedicina (1981-2005). Revista Española de Documentación Científica, 31, 344-365. doi:10.3989/redc.2008.v31.i3.433.

González-Alcaide, G., Bolaños-Pizarro, M., Navarro Molina, C., De Granda-Orive, J. I., Aleixandre-Benavent, R. y Valderrama-Zurián, J. C. (2008b). Análisis de la producción científica española sobre abuso de sustancias en función de la colaboración entre disciplinas. Adicciones, 20, 337-346.

González-Alcaide, G., Valderrama-Zurián, J. C. y Aleixandre-Benavent, R. (2012). Análisis del proceso de internacionalización de la investigación española en ciencia y tecnología (1980-2007). Revista Española de Documentación Científica, 35, 94-118. doi:10.3989/redc.2012.1.847.

Guardiola, E., Sánchez-Carbonell, X., Beranuy, M. y Bellés, A. (2006). La producción científica española sobre dependencia de drogas en el contexto de la Unión Europea: 1976-2000. Adicciones, 18, 119-133.

Huang, M. H y Chang, C. P. (2014). Detecting research fronts in OLED field using bibliographic coupling with sliding window. Scientometrics, 98, 1721-1744. doi:10.1007/s11192-013-1126-1.

Jarneving, B. (2001). The cognitive structure of current cardiovascular research. Scientometrics, 50, 365-389. doi: 10.1023/A:1010546312154.

Jarneving, B. (2005). A comparison of two bibliometric methods for mapping of the research front. Scientometrics, 65, 245-263.

Jarneving, B. (2007a). Bibliographic coupling and its application to research-front and other core docu- 
ments. Journal of Informetrics, 1, 287-307. doi: 10.1016/j. joi.2007.07.004.

Jarneving, B. (2007b). Complete graphs and bibliographic coupling: A test of the applicability of bibliographic coupling for the identification of cognitive cores on the field level. Journal of Informetrics, 1, 338-356. doi: 10.1016/j.joi.2007.08.001.

Jarneving, B. (2013). The impact of core documents: a citation analysis of the 2003 Science Citation Index core-document population. En: Proceedings of the ISSI 2013 Viena. 14th International Society of Scientometrics and Informetrics Conference, Viena, Austria, 15th to 19th July 2013 (pp. 955-965). Viena, Austria: Austrian Institute of Technology.

Kessler, M. M. (1963). Bibliographic coupling between scientific papers. American Documentation, 14, 10-25. doi: 10.1002/asi.5090140103.

Kuusi, O. y Meyer, M. (2007). Anticipating technological breakthroughs: using bibliographic coupling to explore the nanotubes paradigm. Scientometrics, 70, 759-777. doi: 10.1007/s11192-007-0311-5.

Liu, M. (1993). Progress in Documentation. The complexities of citation practice: a review of citation studies. Journal of Documentation, 49, 370-408. doi: 10.1108/ eb026920.

Long, J. C., Cunningham, F. C. y Braithwaite, J. (2012). Network structure and the role of key players in a translational cancer research network: a study protocol. BMJ Open, 2, e001434. doi: 10.1136/bmjopen-2012-001434.

MacRoberts, M. H. y MacRoberts, B. R. (1996). Problems of citation analysis. Scientometrics, 36, 435-344. doi: 10.1007/BF02129604.

Meyer, M., Libaers, D., Thijs, B., Grant, K., Glänzel, W. y Debackere, K. (2014). Origin and Emergence of Entrepreneurship as a Research Field. Scientometrics, 98, 473485. doi: 10.1007/s11192-013-1021-9.

Nicolaisen, J. y Frandsen, T. F. (2012) . Consensus formation in science modeled by aggregated bibliographic coupling. Journal of Informetrics, 6, 276-284. doi: 10.1016/j. joi.2011.08.001.

Osca Lluch, J. (2012). Productividad y colaboración científica desde una perspectiva de género en la Revista Española de Drogodependencias. Revista Española de Drogodependencias, 37, 9-21.

Porcel Torrens, A., Castellano-Gómez, M., Valderrama-Zurián, J. C., Aleixandre-Benavent, A. y Choren, S. (2003). Análisis de citas en la revista Adicciones. Adicciones, 15, 309-319.

Sánchez-Carbonell, X., Guardiola, E., Bellés, A. y Beranuy, M. (2005). European Union scientific production on alcohol and drug misuse (1976-2000). Addiction, 100, 1166-1174. doi: 10.1111/j.1360-0443.2005.01135.x.

Small, H. (1973). Co-citation in the scientific literature: a new measure of the relationship between two docu- ments. Journal of the American Society for Information Science, 24, 265-269. doi: 10.1002/asi.4630240406.

Small, H. (2009). Critical thresholds for co-citation clusters and emergence of the giant component. Journal of Informetrics, 3, 332-340. doi: 10.1016/j.joi.2009.05.001.

Upham, S. P. y Small, H. (2010). Emerging research fronts in science and technology: patterns of new knowledge development. Scientometrics, 83, 15-38. doi: 10.1007/ s11192-009-0051-9.

Vogel, R. y Güttel, W. H. (2013). The dynamic capability view in Strategic Management: a bibliometric review. International Journal of Management Reviews, 15, 426-446. doi: 10.1111/ijmr.12000.

Youtie, J., Kay, L. y Melkers, J. (2013). Bibliographic coupling and network analysis to assess knowledge coalescence in a research center environment. Research Evaluation, 22, 145-156. doi: 10.1093/reseval/rvt002.

Yuan, Y., Gretzel, U. y Tseng, Y. H. (2014). Revealing the nature of contemporary tourism research: Extracting common subject areas through bibliographic coupling. International Journal of Tourism Research. En prensa. doi: 10.1002/jtr.2004. 